\section{S. Natsiavas}

Department of Mechanical Engineering, Aristotle University of Thessalonki, 54006 Thessaloniki, Greece

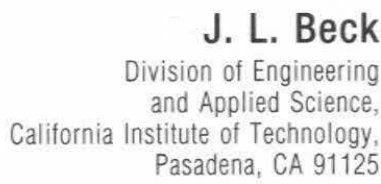

\title{
Almost Classically Damped Continuous Linear Systems
}

The dynamic response of a general class of continuous linear vibrating systems is analyzed which possess damping properties close to those resulting in classical (uncoupled) normal modes. First, conditions are given for the existence of classical modes of vibration in a continuous linear system, with special attention being paid to the boundary conditions. Regular perturbation expansions in terms of undamped modeshapes are then utilized for analyzing the eigenproblem as well as the vibration response of almost classically damped systems. The analysis is based on a proper splitting of the damping operators in both the field equations and the boundary conditions. The main advantage of this approach is that it allows application of standard modal analysis methodologies so that the problem is reduced to that of finding the frequencies and mode shapes of the corresponding undamped system. The approach is illustrated by two simple examples involving rod and beam vibrations.

\section{Introduction}

In many instances, the dynamic response of a vibrating system can be well predicted by a linear model. When damping effects are neglected, the equations of motion of these systems can be reduced to a set of uncoupled ordinary differential equations, each representing a single-degree-of-freedom linear oscillator. However, introduction of linear viscous damping allows this uncoupling only if the damping distribution bears a special relationship with the mass and stiffness distributions of the system (Caughey, 1960; Caughey and O'Kelly, 1965). If it does, the system is often said to be "classically damped" and to possess "classical normal modes." The terminology "proportional damping' 'is also used but this is easily confused with the very special case of Rayleigh damping (Rayleigh, 1945), where the damping distribution is a linear combination of the mass and stiffness distributions.

The existence of classical normal modes provides a simple physical interpretation of the behavior of a vibrating system as a linear superposition of characteristic modes of vibration of the system. It also leads to computational advantages when only a few modes of vibration contribute to the response of the system because of a limited bandwidth excitation. When the conditions for classical modes are not met exactly (e.g., Natsiavas, 1993), the system response may be obtained by one of several different methods: by direct numerical integration of the equations of motion; or by properly converting these equations to first-order state-space form (e.g., Foss, 1958; Meirovitch, 1967); or by approximating the damping in some way so that classical modes exist (Bellos and Inman, 1989).

The present work focuses on the response of linear continuous systems which do not possess classical normal modes, but the damping properties are close to those meeting the conditions leading to normal modes. These systems are called "almost classically damped"' and are of practical significance. As a result, various aspects of their response have already been examined by others, but with emphasis placed on discrete systems. For example, Chung and Lee (1986) extended a perturbation

Contributed by the Applied Mechanics Division of THE AMERICAN SOCIETY OF MECHANICAL ENGINEERS for publication in the ASME JOURNAL OF APPLIED MECHANICS

Discussion on the paper should be addressed to the Technical Editor, Professor Lewis T. Wheeler, Department of Mechanical Engineering, University of Houston, Houston, TX 77204-4792, and will be accepted until four months after fina publication of the paper itself in the ASME JOURNAL OF APPLIED MECHANICS.

Manuscript received by the ASME Applied Mechanics Division, July 22, 1997; final revision, Apr. 3, 1998. Associate Technical Editor: N. C. Perkins. methodology developed earlier by Meirovitch and Ryland (1979) in order to obtain the eigensolution of these discrete systems. Also, Cronin (1976) presented a perturbation analysis for the response of such systems under harmonic excitation, while Udwadia and Esfandiari (1990) and Udwadia and Kumar (1994) presented an iterative approach for general forcing functions. Other relevant work in discrete systems include the contributions of Knowles (1985), Nicholson (1987), Shahruz and Ma (1988), and Natsiavas and Beck (1994). Various aspects of damped continuous linear systems have also been examined (e.g., Caughey and O'Kelly, 1965; Pan, 1966; Plaut and Infante, 1972; Inman and Andry, 1982; Bergman and Nicholson, 1985; Yang, 1996). More references and information about damping effects can also be found in the books by Snowdon (1968) and Nashif et al. (1985).

The objectives of the present work are, first, to present conditions for the existence of classical modes for continuous linear vibrating systems and, second, to present a perturbation approach for the approximate solution of almost classically damped continuous linear systems under arbitrary forcing functions. For the first objective, the work of Caughey and O'Kelly (1965) is extended by being specific about the concept of "compatible"' internal and boundary operators for both the stiffness and damping characteristics. For the second objective, an analysis is developed, based on a proper decomposition of the internal and boundary damping operators. The approach uses classical modal analysis so that the solution is obtained by utilizing only the real modes and frequencies of the corresponding undamped system. This is especially efficient in cases where the solution of a classically damped system has been obtained and the effect of small changes in the damping distribution is sought. Finally, the approach is illustrated by two simple examples.

\section{Problem Statement and Definitions}

Boundary Value Problem for Forced Vibrations. The forced vibration behavior of a linear elastic viscously damped continuous body, such as a stretched rod, a torsional shaft, a bending beam, a membrane or a plate, is commonly governed by an equation of motion of the form

$$
m(\mathbf{r}) \ddot{u}+L_{1} \dot{u}+L_{2} u=f(\mathbf{r}, t) \quad \forall \mathbf{r} \in S
$$

where the body occupies $\bar{S}=S \cup \partial S$. Here $S$ is a bounded domain in $\mathbb{R}^{n_{s}}\left(n_{s}=1,2\right.$ or 3$)$ with boundary $\partial S$. Typically, $u(\mathbf{r}, t)$ is a (generalized) displacement at position $\mathbf{r}$ in the body 
at time $t$, while $\dot{u}(\mathbf{r}, t)$ and $\ddot{u}(\mathbf{r}, t)$ denote the corresponding velocity and acceleration; $m(\mathbf{r})$ is the mass density of the body; $f(\mathbf{r}, t)$ is an external body force and $L_{1}$ and $L_{2}$ are time-invariant linear differential spatial operators of orders $n_{1}$ and $n_{2}$, respectively (typically $n_{1} \leq n_{2}$ ), which model the internal damping forces and elastic restoring forces, respectively, within the body.

The appropriate boundary conditions for (1) are of the form

$$
\begin{gathered}
D_{N}^{(i-1)} u=O \quad \forall \mathbf{r} \in \partial S_{i} \\
\text { and } \quad B_{i} u+C_{i} \dot{u}=g_{i}(\mathbf{r}, t) \quad \forall \mathbf{r} \in \partial S_{i}^{\prime}
\end{gathered}
$$

for $i=1, \ldots, n_{b}\left(=1 / 2 n_{2}\right.$, typically $)$, expressing the fact that for each $i$, on some parts of the boundary $\left(\partial S_{i}\right)$, a geometric condition will hold, while on the other parts $\left(\partial S_{i}^{\prime}\right)$, a generalized force condition will hold (so $\partial S_{i} \cup \partial S_{i}^{\prime}=\partial S, \partial S_{i} \cap$ $\left.\partial S_{i}^{\prime}=\phi\right) . D_{N}^{(i)}$ denotes the derivative of order $i$ normal to the boundary surface at $\mathbf{r} \in \partial S$, with $D_{N}^{(0)} u$ being simply $u$ itself. Each $B_{i}$ and $C_{i}$ is a time-invariant linear differential spatial operator of order $\left(n_{2}-i\right)$ and $\left(n_{1}-i\right)$, respectively, which models the deformation-induced internal and external generalized restoring forces and damping forces, respectively, at the boundary. Each $g_{i}(\mathbf{r}, t)$ represents the resultant of any external generalized forces applied at the boundary of the body at time $t$.

An important additional assumption is that the $B_{i}$ and $C_{i}$ are compatible boundary operators for $L_{2}$ and $L_{1}$, respectively, in the sense that they are related through "integration by parts", (or a generalized Green's formula) by

$$
\begin{aligned}
\left\langle L_{1} \psi, \phi\right\rangle-\left\langle\psi, L_{1} \phi\right\rangle=\sum_{i=1}^{n_{b}}\left[\left(D_{N}^{(i-1)} \psi\right.\right. & \left., C_{i} \phi\right) \\
& \left.-\left(C_{i} \psi, D_{N}^{(i-1)} \phi\right)\right]
\end{aligned}
$$

and

$$
\left\langle L_{2} \psi, \phi\right\rangle-\left\langle\psi, L_{2} \phi\right\rangle=\sum_{i=1}^{n_{b}}\left[\left(D_{N}^{(i-1)} \psi, B_{i} \phi\right)-\left(B_{i} \psi, D_{N}^{(i-1)} \phi\right)\right]
$$

for all $\psi, \phi \in V$, a linear space of suitably smooth functions on $\bar{S}$, such as $C^{n_{3}}(S) \cap C^{n_{3}-1}(\bar{S})$, where $n_{3}=\max \left(n_{1}, n_{2}\right)$, and which satisfy the geometric boundary conditions in (2). Here $C^{n_{3}}(S)$ denotes the set of functions on $S$ which are continuously differentiable up to order $n_{3}$. The body and boundary inner products in (3) and (4) are defined by

$$
\langle\psi, \phi\rangle=\int_{S} \psi(\mathbf{r}) \phi(\mathbf{r}) d \mathbf{r}
$$

and

$$
(\psi, \phi)=\int_{\partial S} \psi(\mathbf{r}) \phi(\mathbf{r}) d S(\mathbf{r}) .
$$

The compatibility conditions (3) and (4) are types of reciprocity principles which are to hold in the absence of external applied body and boundary forces. Condition (3) can be interpreted as stating that the virtual power from the virtual velocity $\phi$ together with the body and boundary damping forces set up by the virtual velocity $\psi$ must be equal to the virtual power with the roles of $\phi$ and $\psi$ swapped. Similarly, condition (4) can be interpreted as stating that the virtual work from the virtual displacement $\phi$ together with the body and boundary restoring forces set up by the virtual displacement $\psi$ must be equal to the virtual work with the roles of $\phi$ and $\psi$ swapped.

Equations (1) and (2), together with specified initial conditions $u(\mathbf{r}, 0)$ and $\dot{u}(\mathbf{r}, 0)$ on $S$, are assumed to give a wellposed boundary value problem on $S \times[0, \infty)$ with solution $u$ $\in V, \forall t \in[0, \infty)$. The goal is to find an approximate solution when the system is almost classically damped.
Undamped System. We start with the undamped homogeneous version of Eqs. (1) and (2) where $L_{1}$ and $f$ are zero on $S$ and each $C_{i}$ and $g_{i}$ is zero on $\partial S_{i}^{\prime}$. We define a linear subspace $U_{0}$ of $V$ consisting of all those functions in $V$ which satisfy this simplified version of the boundary conditions (2). From Eq. (4),

$$
\left\langle L_{2} \psi, \phi\right\rangle=\left\langle\psi, L_{2} \phi\right\rangle \quad \forall \psi, \phi \in U_{0}
$$

so that $L_{2}$ is a self-adjoint linear operator on $U_{0}$ with respect to the inner product defined by (5). We assume, therefore, as is typical for vibration problems, that $m^{-1} L_{2}$ has a discrete spectrum of distinct positive eigenvalues $\left\{\omega_{n}^{2}: n \in \mathbf{Z}^{+}\right\}$and a corresponding complete set of orthonormal real eigenfunctions $\left\{\phi_{n}(\mathbf{r}): n \in \mathbf{Z}^{+}\right\}$in $U_{0}$ so that $\forall \psi \in V$ :

$$
\psi(\mathbf{r})=\sum_{n=1}^{\infty}\left\langle\psi, m \phi_{n}\right\rangle \phi_{n}(\mathbf{r}) \quad \forall \mathbf{r} \in S
$$

where the orthonormality guaranteed by the self-adjointness of $L_{2}$ gives.

$$
\left\langle\phi_{n}, m \phi_{p}\right\rangle=\delta_{n p},
$$

the Kronecker delta, and

$$
\begin{gathered}
L_{2} \phi_{n}=\omega_{n}^{2} m \phi_{n} \text { on } S, \\
D_{N}^{(i-1)} \phi_{n}=0 \quad \text { on } \partial S_{i} \quad \text { and } B_{i} \phi_{n}=0 \\
\text { on } \partial S_{i}^{\prime} \quad \forall i=1, \ldots, n_{b} .
\end{gathered}
$$

In general, the eigenfunction expansion in (7) will only converge to $\psi$ on the boundary $\partial S$ if $\psi$ and the $\phi_{n}$ satisfy the same boundary conditions (e.g., Courant and Hilbert, 1989, Chapter $5)$. In this case, it is typical to have uniform convergence on $\bar{S}$ and no Gibbs phenomenon appears near the boundary $\partial S$. This behavior is advantageous in applications because then the eigenfunction series converges faster, that is, a smaller number of terms is required in (7) to give an approximation of the function $\psi$ to within a specified accuracy.

Almost Classically Damped System. We now consider two special cases of the full damped linear system given by Eqs. (1) and (2):

Definition: The damped system is classically damped if the body and boundary damping operators satisfy

$$
L_{1} \phi_{n}=\beta_{n} m \phi_{n} \quad \text { on } S
$$

for some constants $\beta_{n} \geq 0$, and

$$
C_{i} \phi_{n}=0 \text { on } \partial S_{i}^{\prime} \quad \forall i=1, \ldots, n_{b} .
$$

This means that the eigenfunctions $\phi_{n}, n \in \mathbf{Z}^{+}$, of $L_{2}$ on $U_{0}$ are also eigenfunctions of $L_{1}$ on $U_{0}$. For this to be possible, it is necessary that $L_{1}$ be a positive semidefinite self-adjoint linear operator on $U_{0}$ and that $m^{-1} L_{1}$ and $m^{-1} L_{2}$ commute on $U_{0}$. These conditions are also sufficient for (11) to hold (Caughey and O'Kelly, 1965). We shall hereafter denote the body and boundary damping operators for a classically damped system by $L_{10}$ and $C_{i 0}, i=1, \ldots, n_{b}$, respectively.

The eigenfunctions of a classically damped system are identical to the undamped eigenfunctions, $\phi_{n}(\mathbf{r}), n \in \mathbf{Z}^{+}$. To show this, let $\hat{\phi}_{n}$ denote an eigenfunction corresponding to eigenvalue $\rho_{n}$, then $\hat{\phi}_{n}(\mathbf{r}) e^{\rho_{n} t}$ is a solution of Eqs. (1) and (2) with $f=0$ on $S$ and each $g_{i}=0$ on $\partial S_{i}^{\prime}$, so

$$
\left(\rho_{n}^{2} m+\rho_{n} L_{10}+L_{2}\right) \hat{\phi}_{n}=0 \text { on } S
$$

and $\forall i=1, \ldots n_{b}$,

$$
D_{N}^{(i-1)} \hat{\phi}_{n}=0 \text { on } \partial S_{i}, \quad\left(B_{i}+\rho_{n} C_{i 0}\right) \hat{\phi}_{n}=0 \text { on } \partial S_{i}^{\prime} .
$$

In view of Eqs. (9)-(12), $\hat{\phi}_{n}=\phi_{n}$ is the solution if $\rho_{n}$ satisfies 


$$
\rho_{n}^{2}+\beta_{n} \rho_{n}+\omega_{n}^{2}=0 .
$$

The two roots of (13) corresponding to a given $\phi_{n}$ are a complex conjugate pair in the underdamped case, that is, when $\beta_{n}<$ $2 \omega_{n}$.

Definition. The damped system defined by equations (1) and (2) is almost classically damped if

$$
L_{1}=L_{10}+\epsilon L_{11}, \quad C_{i}=C_{i 0}+\epsilon C_{i 1} \quad \forall i=1, \ldots, n_{b}
$$

where $\epsilon$ is a small parameter and $L_{10}$ and the $C_{i 0}$ are the classically damped operators defined by (11) and (12), respectively.

In this case, the damping operators are perturbed slightly from the classically damped case, so it is possible to analyze the system using an approximate perturbation approach, as shown in the subsequent sections. Although $\epsilon$ is assumed to be small, the underlying classical damping need not be small. It is assumed that the compatibility condition ( 3 ) is satisfied by the classical-damping operators $L_{10}$ and $C_{i 0}, \forall i$, and so by linearity, the damping operators $L_{11}$ and $C_{i 1}, \forall i$, also satisfy this condition.

\section{Approximate Solution of the Eigenproblem}

Let $\hat{\phi}_{p}, p \in \mathbf{Z}^{+}$, be an eigenfunction of the almost classically damped system, with corresponding eigenvalue $s_{p}$, then

$$
u_{p}(\mathbf{r}, t)=\hat{\phi}_{p}(\mathbf{r}) e^{s_{p} t}
$$

is a solution of Eqs. (1) and (2) with $f=0$ on $S$ and each $g_{i}$ $=0$ on $\partial S_{i}^{\prime}$, so

$$
\left(s_{p}^{2} m+s_{p} L_{1}+L_{2}\right) \hat{\phi}_{p}=0 \text { on } S
$$

and $\forall i=1, \ldots n_{b}$

$$
\begin{gathered}
D_{N}^{(i-1)} \hat{\phi}_{p}=0 \text { on } \partial S_{i} \quad \text { and } \\
\left(B_{i}+s_{p} C_{i}\right) \hat{\phi}_{p}=0 \text { on } \partial S_{i}^{\prime} .
\end{gathered}
$$

Since $\epsilon$ is small in (14), we expect that $\hat{\phi}_{p}$ and $s_{p}$ will remain close to the eigenquantities $\phi_{p}$ and $\rho_{p}$ of the classically damped system, which satisfy Eqs. (9) through (13) when $L_{1}$ and $C_{i}$ are replaced by $L_{10}$ and $C_{i 0}$ in Eqs. (11) and (12). We therefore use the asymptotic expansions (Erdelyi, 1956)

$$
\begin{aligned}
\hat{\phi}_{p}(\mathbf{r}) & \sim \phi_{p}(\mathbf{r})+\epsilon \chi_{p}(\mathbf{r})+\epsilon^{2} \psi_{p}+\ldots \\
s_{p} & \sim \rho_{p}+\epsilon \sigma_{p}+\epsilon^{2} \tau_{p}+\ldots
\end{aligned}
$$

In the underdamped case, each $s_{p}$ is complex-valued, and so, therefore, are the quantities in these expansions, except $\phi_{p}$. Corresponding to each complex conjugate eigenvalue pair, $\rho_{p}$ and $\bar{\rho}_{p}$, for the underlying classically damped system, there are perturbed eigenvalues $s_{p}$ and $\bar{s}_{p}$, respectively, with associated eigenfunctions $\hat{\phi}_{p}(\mathbf{r})$ and $\bar{\phi}_{p}(\mathbf{r})$. It is therefore only necessary to determine the perturbed eigenvalue and eigenfunction for one of the two complex roots of Eq. (13). In the overdamped case, however, it is necessary to determine separately a perturbed eigenvalue and eigenfunction for each of the real-valued roots of Eq. (13).

For suitably small $\epsilon$, the second-order expansions are expected to be sufficient to capture the essential features of the eigenquantities. By substituting (17) and (18) into (15) and (16), taking Eqs. (9)-(14) into account, we get from the firstorder terms in $\epsilon$

$$
\mathcal{L}_{p} \chi_{p}=\zeta_{p} \equiv-m \sigma_{p}\left(2 \rho_{p}+\beta_{p}\right) \phi_{p}-\rho_{p} L_{11} \phi_{p} \quad \text { on } S
$$

and $\forall i=1, \ldots, n_{b}$,

$$
\begin{gathered}
D_{N}^{(i-1)} \chi_{p}=0 \quad \text { on } \partial S_{i}, \\
\mathcal{B}_{i p} \chi_{p}=\zeta_{i p} \equiv-\rho_{p} C_{i 1} \phi_{p} \quad \text { on } \partial S_{i}^{\prime} .
\end{gathered}
$$

Likewise, the second-order terms in $\epsilon$ give

$$
\begin{aligned}
\mathcal{L}_{p} \psi_{p}= & \eta_{p} \equiv-\left(2 m \rho_{p} \sigma_{p}+\sigma_{p} L_{10}+\rho_{p} L_{11}\right) \chi_{p} \\
& -m\left(\sigma_{p}^{2}+2 \rho_{p} \tau_{p}+\beta_{p} \tau_{p}\right) \phi_{p}-\sigma_{p} L_{11} \phi_{p} \text { on } S
\end{aligned}
$$

and $\forall i=1, \ldots, n_{b}$,

$D_{N}^{(i-1)} \psi_{p}=0$ on $\partial S_{i}$,

$\mathcal{B}_{i p} \psi_{p}=\eta_{i p} \equiv-\left(\sigma_{p} C_{i 0}+\rho_{p} C_{i 1}\right) \chi_{p}-\sigma_{p} C_{i 1} \phi_{p} \quad$ on $\partial S_{i}^{\prime}$.

In these equations, the body and boundary operators $\mathcal{L}_{p}$ and $\mathcal{B}_{i p}$ are defined by

$$
\begin{gathered}
\mathcal{L}_{p} \equiv \rho_{p}^{2} m+\rho_{p} L_{10}+L_{2} \\
\mathcal{B}_{i p} \equiv B_{i}+\rho_{p} C_{i 0}, \quad \forall i=1, \ldots, n_{b} .
\end{gathered}
$$

Using Eqs. (9)-(13), we find

$$
\begin{gathered}
\mathcal{L}_{p} \phi_{q}=m \pi_{p q} \phi_{q} \text { on } S \\
\mathcal{B}_{i p} \phi_{q}=0 \text { on } \partial S_{i}^{\prime}, \quad \forall i=1, \ldots, n_{b}
\end{gathered}
$$

where $\pi_{p q}$ is defined by

$$
\pi_{p q}=\rho_{p}^{2}+\rho_{p} \beta_{q}+\omega_{q}^{2}
$$

so that $\pi_{p p}=0$, from Eq. (13). Using the compatibility Eqs. (3) and (4), we can show that $\forall \psi, \phi \in V$,

$$
\begin{aligned}
\left\langle\mathcal{L}_{p} \psi, \phi\right\rangle & -\left\langle\psi, \mathcal{L}_{p} \phi\right\rangle \\
& =\sum_{i=1}^{n_{b}}\left[\left(D_{N}^{(i-1)} \psi, \mathcal{B}_{i p} \phi\right)-\left(\mathcal{B}_{i p} \psi, D_{N}^{(i-1)} \phi\right)\right] .
\end{aligned}
$$

To determine the first-order terms $\chi_{p}$ and $\sigma_{p}$ in (17) and (18) from Eqs. (19) and (20) in a way that avoids convergence problems on the boundary, we first employ the decomposition

$$
\chi_{p}(\mathbf{r})=\hat{\chi}_{p}(\mathbf{r})+\mu_{p}(\mathbf{r}) \text { on } \bar{S}
$$

where $\mu_{p}$ is chosen as any function in $V$ which satisfies the following boundary conditions for each $i=1, \ldots, n_{b}$,

$$
D_{N}^{(i-1)} \mu_{p}=0 \quad \text { on } \partial S_{i}, \quad \mathcal{B}_{i p} \mu_{p}=\zeta_{i p} \quad \text { on } \partial S_{i}^{\prime} .
$$

Then, from Eq. (19), $\hat{\chi}_{p} \in V$ is found to satisfy the transformed equations

$$
\mathcal{L}_{p} \hat{\chi}_{p}=\zeta_{p}-\mathcal{L}_{p} \mu_{p} \text { on } S
$$

and $\forall i=1, \ldots, n_{b}$,

$$
D_{N}^{(i-1)} \hat{\chi}_{p}=0 \quad \text { on } \partial S_{i}, \quad \mathcal{B}_{i p} \hat{\chi}_{p}=0 \quad \text { on } \partial S_{i}^{\prime} .
$$

Since $\hat{\chi}_{p}$ satisfies the same boundary conditions as the complete orthonormal set $\left\{\phi_{q}(\mathbf{r}), q \in \mathbf{Z}^{+}\right\}$(see Eqs. (10) and (25)), its eigenfunction expansion

$$
\hat{\chi}_{p}(\mathbf{r})=\sum_{q=1}^{\infty} \alpha_{p q} \phi_{q}(\mathbf{r}) \quad \text { on } \bar{S}
$$

where

$$
\alpha_{p q}=\left\langle\hat{\chi}_{p}, m \phi_{q}\right\rangle
$$

can also be expected to converge on the boundary $\partial S$ (e.g., Courant and Hilbert, 1989, Chapter 5). To determine $\chi_{p}$, it remains to determine the $\alpha_{p q}, q \in \mathbf{Z}^{+}$.

Using Eqs. (25) - (27) and (29)-(31), we get

$$
\begin{gathered}
\left\langle\mathcal{L}_{p} \hat{\chi}_{p}, \phi_{q}\right\rangle=\pi_{p q} \alpha_{p q} \\
\left\langle\mathcal{L}_{p} \mu_{p}, \phi_{q}\right\rangle=\pi_{p q} \mu_{p q}+\rho_{p} \hat{\zeta}_{p q}
\end{gathered}
$$

if we define 


$$
\begin{gathered}
\mu_{p q}=\left\langle\mu_{p}, m \phi_{q}\right\rangle \\
\hat{\zeta}_{p q}=\sum_{i=1}^{n_{b}}\left(C_{i 1} \phi_{p}, D_{N}^{(i-1)} \phi_{q}\right)_{\partial S_{i}} .
\end{gathered}
$$

But from Eq. (19)

$$
\begin{aligned}
\left\langle\mathcal{L}_{p} \chi_{p}, \phi_{q}\right\rangle & =\left\langle\zeta_{p}, \phi_{q}\right\rangle \\
& =-\sigma_{p}\left(2 \rho_{p}+\beta_{p}\right) \delta_{p q}-\rho_{p} \phi_{p q}
\end{aligned}
$$

if we define

$$
\phi_{p q}=\left\langle L_{11} \phi_{p}, \phi_{q}\right\rangle .
$$

Using Eq. (30) to relate Eqs. (34), (35), and (38),

$$
\pi_{p q} \alpha_{p q}=-\sigma_{p}\left(2 \rho_{p}+\beta_{p}\right) \delta_{p q}-\rho_{p} \phi_{p q}-\pi_{p q} \mu_{p q}-\rho_{p} \hat{\zeta}_{p q} \text {. }
$$

When $p=q$, this equation determines the first-order eigenvalue correction constant

$$
\sigma_{p}=-\rho_{p} \frac{\hat{\zeta}_{p p}+\phi_{p p}}{2 \rho_{p}+\beta_{p}},
$$

while for $p \neq q$, it determines the Fourier coefficients

$$
\alpha_{p q}=-\rho_{p}\left(\hat{\zeta}_{p q}+\phi_{p q}\right) / \pi_{p q}-\mu_{p q} .
$$

The coefficients $\alpha_{p p}$ will be determined later.

Next, in order to determine the second-order terms $\psi_{p}$ and $\tau_{p}$ in (17) and (18) from Eqs. (21) and (22), we again employ a decomposition

$$
\psi_{p}(\mathbf{r})=\hat{\psi}_{p}(\mathbf{r})+v_{p}(\mathbf{r}) \text { on } \bar{S}
$$

where $v_{p}$ is chosen as any function in $V$ which satisfies for each $i=1, \ldots, n_{b}$, the conditions

$$
D_{N}^{(i-1)} v_{p}=0 \quad \text { on } \partial S_{i}, \quad \mathcal{B}_{i p} v_{p}=\eta_{i p} \quad \text { on } \partial S_{i}^{\prime} .
$$

Then, from Eq. (21), $\hat{\psi}_{p} \in V$ satisfies

$$
\mathcal{L}_{p} \hat{\psi}_{p}=\eta_{p}-\mathcal{L}_{p} v_{p} \text { on } S
$$

and $\forall i=1, \ldots, n_{b}$,

$$
D_{N}^{(i-1)} \hat{\psi}_{p}=0 \quad \text { on } \partial S_{i}, \quad \mathcal{B}_{i p} \hat{\psi}_{p}=0 \quad \text { on } \partial S_{i}^{\prime} .
$$

Therefore

$$
\hat{\psi}_{p}(\mathbf{r})=\sum_{q=1}^{\infty} \beta_{p q} \phi_{q}(\mathbf{r}) \quad \text { on } \bar{S}
$$

where

$$
\beta_{p q}=\left\langle\hat{\psi}_{p}, m \phi_{q}\right\rangle .
$$

Using Eqs. (25) - (27) again and following a similar argument as before, we find

$$
\begin{gathered}
\left\langle\mathcal{L}_{p} \hat{\psi}_{p}, \phi_{q}\right\rangle=\pi_{p q} \beta_{p q} \\
\left\langle\mathcal{L}_{p} v_{p}, \phi_{q}\right\rangle=\pi_{p q} v_{p q}-\hat{\eta}_{p q},
\end{gathered}
$$

if we define

$$
\begin{gathered}
v_{p q}=\left\langle v_{p}, m \phi_{q}\right\rangle \\
\hat{\eta}_{p q}=\sum_{i=1}^{n_{b}}\left(\eta_{i p}, D_{N}^{(i-1)} \phi_{q}\right)_{\partial s_{i}^{\prime}} .
\end{gathered}
$$

But from Eq. (21),

$$
\begin{aligned}
\left\langle\mathcal{L}_{p} \psi_{p}, \phi_{q}\right\rangle & =\left\langle\eta_{p}, \phi_{q}\right\rangle \\
& =-\left(\sigma_{p}^{2}+2 \rho_{p} \tau_{p}+\beta_{p} \tau_{p}\right) \delta_{p q}-\theta_{p q},
\end{aligned}
$$

where we define

$$
\theta_{p q}=\sigma_{p} \phi_{p q}+\left\langle\left(2 m \rho_{p} \sigma_{p}+\sigma_{p} L_{10}+\rho_{p} L_{11}\right) \chi_{p}, \phi_{q}\right\rangle .
$$

Simplified expressions for $\hat{\eta}_{p q}$ and $\theta_{p q}$ defined in Eqs. (52) and (54) are given in Appendix A.

Using Eq. (45) to relate Eqs. (49), (50), and (53),

$$
\begin{aligned}
\pi_{p q} \beta_{p q}=-\left(\sigma_{p}^{2}+2 \rho_{p} \tau_{p}+\beta_{p} \tau_{p}\right) & \delta_{p q} \\
& -\theta_{p q}-\pi_{p q} v_{p q}+\hat{\eta}_{p q} .
\end{aligned}
$$

When $p=q$, this equation determines the second-order eigenvalue correction constant

$$
\tau_{p}=\frac{\hat{\eta}_{p p}-\theta_{p p}-\sigma_{p}^{2}}{2 \rho_{p}+\beta_{p}},
$$

while for $p \neq q$, it determines the Fourier coefficients

$$
\beta_{p q}=\left(\hat{\eta}_{p q}-\theta_{p q}\right) / \pi_{p q}-v_{p q} .
$$

Finally, $\alpha_{p p}$ and $\beta_{p p}$ can be determined by normalizing the eigenfunction $\hat{\phi}_{p}$ so that

$$
\left\langle\hat{\phi}_{p}, m \hat{\phi}_{p}\right\rangle=1 .
$$

Employing Eqs. (8), (17), (28), (32), (33), and (48), the last condition yields

$$
\begin{gathered}
\alpha_{p p}=-\mu_{p p} \\
\beta_{p p}=-v_{p p}+\frac{1}{2} \sum_{q=1}^{\infty}\left(\mu_{p q}^{2}-\epsilon_{p q}^{2}\right)
\end{gathered}
$$

where

$$
\epsilon_{p q}= \begin{cases}0, & p=q \\ \left(\hat{\zeta}_{p q}-\rho_{p} \phi_{p q}\right) / \pi_{p q}, & p \neq q .\end{cases}
$$

In summary, the above analysis gives an asymptotic approximation for the solution of the eigenproblem of almost classically damped continuous linear systems to second order in the small damping parameter $\epsilon$ in Eq. (14). The analysis breaks down when multiple eigenvalues occur in the underlying classically damped system, since then the quantity $\pi_{p p}$ appearing in Eqs. (42) and (57) can be zero for $p \neq q$. A general procedure for treating this case can be found in Courant and Hilbert (1989). The analysis also breaks down at Eqs. (41) and (56), when $2 \rho_{p}+\beta_{p}=0$ for some $p \in \mathbf{Z}^{+}$, which corresponds to a critically damped system, that is, when $\beta_{p}=2 \omega_{\rho}$.

\section{Approximate Solution for Forced Vibrations}

Let $u(\mathbf{r}, t)$ be the general solution of (1) and (2) so that $u$ $\in V$ for each time $t \in[0, \infty)$. We again use an asymptotic expansion in terms of the small damping parameter $\epsilon$ in Eq. (14),

$$
u(\mathbf{r}, t) \sim \sum_{p=0}^{\infty} u_{p}(\mathbf{r}, t) \epsilon^{p} \quad \text { on } \bar{S} \times[0, \infty)
$$

where $u_{0}(\mathbf{r}, t)$ is the solution for the classically damped case $(\epsilon=0)$. This gives a regular perturbation problem which will give a good approximation for the vibration response over some finite time interval, but not as $t \rightarrow \infty$ (Kevorkian and Cole, 1981). Substitution of this expression and Eq. (14) into the field Eq. (1) yields

$$
\mathcal{L} u_{p} \equiv m \ddot{u}_{p}+L_{10} \dot{u}_{p}+L_{2} u_{p}=f_{p}(\mathbf{r}, t) \quad \text { on } S \times(0, \infty)
$$

with

$$
f_{p}= \begin{cases}f(\mathbf{r}, t), & p=0 \\ -L_{11} \dot{u}_{p-1}(\mathbf{r}, t), & p \in \mathbf{Z}^{+} .\end{cases}
$$

Likewise, substitution of (60) and (14) into the boundary conditions ( 2 ) gives $\forall t \in(0, \infty)$ and $\forall i=1, \ldots, n_{b}$ 


$$
\begin{gathered}
D_{N}^{(i-1)} u_{p}=0 \quad \text { on } \partial S_{i}, \\
\mathcal{B}_{i} u_{p} \equiv B_{i} u_{p}+C_{i 0} \dot{u}_{p}=g_{i p}(\mathbf{r}, t) \quad \text { on } \partial S_{i}^{\prime}
\end{gathered}
$$

with

$$
g_{i p}= \begin{cases}g_{i}(\mathbf{r}, t), & p=0 \\ -C_{i 1} \dot{u}_{p-1}(\mathbf{r}, t), & p \in \mathbf{Z}^{+} .\end{cases}
$$

In order for the eigenfunction expansion (7) to be valid over the closure $\bar{S}$ of the domain $S$, the boundary conditions (63) must be put in homogeneous form. For this, we first employ the decomposition

$$
u_{p}(\mathbf{r}, t)=v_{p}(\mathbf{r}, t)+h_{p}(\mathbf{r}, t) \quad \text { on } \bar{S} \times[0, \infty)
$$

where $h_{p}$ is chosen as any real-valued function in $V$ for each $t$, which is twice-differentiable with respect to $t$ almost everywhere on $(0, \infty)$ and satisfies the boundary conditions

$$
\begin{gathered}
D_{N}^{(i-1)} h_{p}=0 \quad \text { on } \partial S_{i}, \\
\mathcal{B}_{i} h_{p}=g_{i p} \text { on } \partial S_{i}^{\prime}, \quad \forall i=1, \ldots, n_{b} .
\end{gathered}
$$

Hence, $v_{p} \in V$ for each $t$, it is twice-differentiable with respect to $t$ almost everywhere on $(0, \infty)$, and it satisfies

$$
\mathcal{L} v_{p}=f_{p}-\mathcal{L} h_{p} \quad \text { on } S
$$

$D_{N}^{(i-1)} v_{p}=0 \quad$ on $\partial S_{i}, \quad \mathcal{B}_{i} v_{p}=0$ on $\partial S_{i}^{\prime}$,

$$
\forall i=1, \ldots, n_{b} .
$$

The functions $v_{p}$ can be expressed in the form

$$
v_{p}(\mathbf{r}, t)=\sum_{n=1}^{\infty} q_{p n}(t) \phi_{n}(\mathbf{r}) \quad \text { on } \bar{S}
$$

where

$$
q_{p n}=\left\langle v_{p}, m \phi_{n}\right\rangle .
$$

We can obtain a differential equation for the $q_{p n}(t)$ by first employing the compatibility conditions (3) and (4):

$$
\begin{aligned}
\left\langle\mathcal{L} v_{p}, \phi_{n}\right\rangle= & \frac{d^{2}}{d t^{2}}\left\langle m v_{p}, \phi_{n}\right\rangle+\frac{d}{d t}\left\langle L_{10} v_{p}, \phi_{n}\right\rangle+\left\langle L_{2} v_{p}, \phi_{n}\right\rangle \\
= & \ddot{q}_{p n}+\frac{d}{d t}\left\{\left\langle v_{p}, L_{10} \phi_{n}\right\rangle+\sum_{i=1}^{n_{b}}\left[\left(D_{N}^{(i-1)} v_{p}, C_{i 0} \phi_{n}\right)\right.\right. \\
& \left.\left.-\left(C_{i 0} v_{p}, D_{N}^{(i-1)} \phi_{n}\right)\right]\right\} \\
+ & \left\{\left\langle v_{p}, L_{2} \phi_{n}\right\rangle+\sum_{i=1}^{n_{b}}\left(D_{N}^{(i-1)} v_{p}, B_{i} \phi_{n}\right)-\left(B_{i} v_{p}, D_{N}^{(i-1)} \phi_{n}\right)\right\} .
\end{aligned}
$$

By using Eqs. (9) to (12), (68), and (70), this expression can be reduced to

$$
\left\langle\mathcal{L} v_{p}, \phi_{n}\right\rangle=\ddot{q}_{p n}+\beta_{n} \dot{q}_{p n}+\omega_{n}^{2} q_{p n} .
$$

In a similar fashion, except that Eq. (66) is used in place of (68), it can be shown that

$$
\left\langle\mathcal{L} h_{p}, \phi_{n}\right\rangle=\ddot{h}_{p n}+\beta_{n} \dot{h}_{p n}+\omega_{n}^{2} h_{p n}-\hat{g}_{p n}(t)
$$

where we define

$$
\begin{gathered}
h_{p n}=\left\langle h_{p}, m \phi_{n}\right\rangle \\
\hat{g}_{p n}=\sum_{i=1}^{n_{b}}\left(g_{i p}, D_{N}^{(i-1)} \phi_{n}\right)_{\partial S_{i}} .
\end{gathered}
$$

Finally, define

$$
f_{p n}(t)=\left\langle f_{p}, \phi_{n}\right\rangle .
$$

A differential equation for each $q_{p n}(t)$ can then be obtained from (67), (72), (73), and (76),

$$
\ddot{q}_{p n}+\beta_{n} \dot{q}_{p n}+\omega_{n}^{2} q_{p n}=Q_{p n}(t)
$$

where we define

$$
Q_{p n}(t)=f_{p n}+\hat{g}_{p n}-\left(\ddot{h}_{p n}+\beta_{n} \dot{h}_{p n}+\omega_{n}^{2} h_{p n}\right) .
$$

Here $f_{p n}$ and $\hat{g}_{p n}$ for $p \in \mathbf{Z}^{+}$depend on $\dot{u}_{p-1}$ through Eqs. (62) and (64), but this dependence can be simplified as shown in Appendix B.

To complete the specification of the coefficients $q_{p n}(t)$, the appropriate initial conditions must be given. From Eqs. (60), $(65)$, and (70),

$$
\begin{aligned}
& q_{p n}(0)=\left\langle u_{p}(\mathbf{r}, 0), m \phi_{n}(\mathbf{r})\right\rangle \\
& =\left\{\begin{array}{l}
\left\langle u(\mathbf{r}, 0), m \phi_{n}(\mathbf{r})\right\rangle-\left\langle h_{0}(\mathbf{r}, 0), m \phi_{n}(\mathbf{r})\right\rangle, p=0 \\
-\left\langle h_{p}(\mathbf{r}, 0), m \phi_{n}(\mathbf{r})\right\rangle, p \in \mathbf{Z}^{+}
\end{array}\right. \\
& =\left\{\begin{array}{c}
\left\langle u(\mathbf{r}, 0), m \phi_{n}(\mathbf{r})\right\rangle-\left\langle\dot{h}_{0}(\mathbf{r}, 0), m \phi_{n}(\mathbf{r})\right\rangle, \\
p=0 \\
-\left\langle\dot{h}_{p}(\mathbf{r}, 0), m \phi_{n}(\mathbf{r})\right\rangle, \\
p \in \mathbf{Z}^{+}
\end{array}\right.
\end{aligned}
$$

where, without loss of generality, we can take $h_{p}(\mathbf{r}, 0)$ and $\dot{h}_{p}(\mathbf{r}, 0)$ as zero for $p=0$ and $p \in \mathbf{Z}^{+}$.

In summary, an asymptotic approximation of the solution of the forced vibration problem to, say, second order in $\epsilon$, may be obtained by using the eigenfunction expansion (69) for $p=0$, 1,2 and by taking a sufficient number of terms, $N$, for the desired accuracy. The coefficients in these expansions for the $v_{p}(\mathbf{r}, t)$ are determined by solving the initial-value problem (77) $-(80)$ for $q_{p n}(t)$ for $p=0,1,2$ and $n=1,2, \ldots, N$.

The functions $h_{p}(\mathbf{r}, t), p=0,1,2$, involved in Eqs. (78) and in the construction of the $u_{p}(\mathbf{r}, t)$ from Eq. (65), must be chosen to satisfy the conditions in Eqs. (66). A typical form for these functions can be obtained by generalizing an expression given in Meirovitch (1967) for the undamped problem, namely,

$$
h_{p}(\mathbf{r}, t)=\sum_{i=1}^{n_{b}}\left[a_{i p}(\mathbf{r}) \lambda_{i p}(t)+b_{i p}(\mathbf{r}) \xi_{i p}(t)\right]
$$

where the functions $a_{i p}, \lambda_{i p}, b_{i p}, \xi_{i p}$ are chosen so that $h_{p}$ satisfies conditions (66), as illustrated later. Once the $u_{p}(\mathbf{r}, t), p=0$, 1,2 , are determined, the solution $u(\mathbf{r}, t)$ can be approximated by the sum of the first three terms in Eq. (60).

Note that the approach developed here avoids direct termby-term differentiation of the eigenfunction series for each $u_{p}$ which would be required for $L_{1} u$ and $L_{2} u$. This operation would be invalid here since each $u_{p}$ satisfies different boundary conditions than the eigenfunctions, $\phi_{n}(\mathbf{r})$.

\section{Examples}

To illustrate the approach, two simple examples are given in this section. 


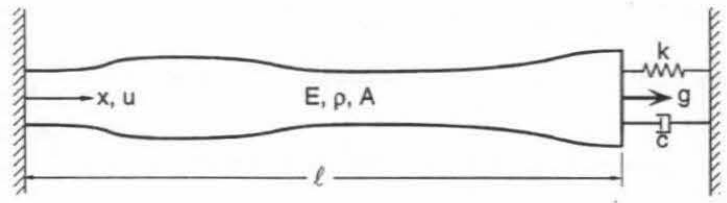

Fig. 1 Rod example

I: Rod Vibrations. Consider the longitudinal vibration of a viscoelastic rod shown in Fig. 1, where Eq. (1) applies with $u(x, t)$ being the longitudinal displacement at time $t$ of a cross section at $x$ and

$$
\begin{gathered}
S=(0, l), \quad \bar{S}=[0, l], \quad \partial S=\{0, l\} \\
m(x)=\rho(x) A(x), f(x, t)
\end{gathered}
$$

$=$ distributed longitudinal load per unit length

$$
\begin{gathered}
L_{1}=\mu_{E}(x)-\frac{\partial}{\partial x}\left[\mu_{I}(x) A(x) \frac{\partial}{\partial x}\right], \\
L_{2}=-\frac{\partial}{\partial x}\left[E(x) A(x) \frac{\partial}{\partial x}\right] .
\end{gathered}
$$

Here $m(x), A(x)$, and $E(x)$ are the mass per unit length, the cross-sectional area and Young's modulus at location $x$ along the rod, while $\mu_{E}(x)$ and $\mu_{I}(x)$ are non-negative viscous damping coefficients. From Fig. 1, the appropriate boundary conditions are geometric at $x=0$ (i.e., $\partial S_{1}$ ) and a force balance at $x=l$ (i.e., $\left.\partial S_{1}^{\prime}\right)$, which give

$$
D_{N}^{(0)} u=u=0 \text { at } x=0, \quad B_{1} u+C_{1} \dot{u}=g(t) \text { at } x=l
$$

where

$$
B_{1}=k+\left.E(l) A(l) \frac{\partial}{\partial x}\right|_{x=l}, \quad C_{1}=c+\left.\mu_{I}(l) A(l) \frac{\partial}{\partial x}\right|_{x=l}
$$

and $g(t)$ represents an applied axial force at $x=l$.

Using integration by parts and the appropriate inner product for this one-dimensional example, we get for all $\psi, \phi \in V \subset$ $C^{2}(0, l) \cap C^{1}[0, l]$ satisfying the geometric boundary condition at $x=0$ :

$$
\begin{aligned}
& \left\langle L_{1} \psi, \phi\right\rangle-\left\langle\psi, L_{1} \phi\right\rangle \\
& =-\int_{0}^{l}\left[\phi \frac{d}{d x}\left(\mu_{1} A \frac{d \psi}{d x}\right)-\psi \frac{d}{d x}\left(\mu_{1} A \frac{d \phi}{d x}\right)\right] d x \\
& =-\left[\mu_{I} A \frac{d \psi}{d x} \phi-\mu_{1} A \frac{d \phi}{d x} \psi\right]_{0}^{l} \\
& =\left[\psi\left(c+\mu_{I} A \frac{d}{d x}\right) \phi-\phi\left(c+\mu_{l} A \frac{d}{d x}\right) \psi\right]_{x=l} \\
& =\left[\psi C_{1} \phi-\phi C_{1} \psi\right]_{x=l}=\left(\psi, C_{1} \phi\right)-\left(C_{1} \psi, \phi\right) .
\end{aligned}
$$

According to (3) with $n_{b}=1, C_{1}$ is therefore confirmed to be a compatible boundary operator for $L_{1}$. In a similar manner, $B_{1}$ can be confirmed to be a compatible boundary operator for $L_{2}$, as defined by Eq. (4).

For the undamped problem, the eigenvalues (frequencies) and eigenfunctions (modeshapes) are determined by

$$
-\left[E(x) A(x) \phi_{n}^{\prime}(x)\right]^{\prime}=\omega_{n}^{2} m(x) \phi_{n}(x) \text { in }(0, l)
$$

and

$$
\phi_{n}(0)=0,\left.\quad B_{1} \phi_{n}\right|_{x=l}=E(l) A(l) \phi_{n}^{\prime}(l)+k \phi_{n}(l)=0
$$

where the prime denotes $d / d x$. These equations define a SturmLiouville problem and so there is a countable set of eigenvalues $\left\{\omega_{n}^{2}: n \in \mathbf{Z}^{+}\right\}$and a complete set of orthonormal eigenfunctions $\left\{\phi_{n}: n \in \mathbf{Z}^{+}\right\}$(Courant and Hilbert, 1989, Chapter 5).

For a classically damped system, we require from Eq. (11)

$$
\mu_{E}(x) \phi_{n}(x)-\left[\mu_{l}(x) A(x) \phi_{n}^{\prime}(x)\right]^{\prime}=L_{1} \phi_{n}=\beta_{n} m(x) \phi_{n}(x) .
$$

This will be satisfied, for example, if we choose

$$
\begin{gathered}
\mu_{E}(x)=\alpha m(x), \quad \mu_{I}(x)=\beta E(x), \\
\beta_{n}=\alpha+\beta \omega_{n}^{2}, \quad \forall n \in \mathbf{Z}^{+}
\end{gathered}
$$

where $\alpha$ and $\beta$ are non-negative constants, which gives

$$
L_{10}=\alpha m+\beta L_{2}
$$

as a classical-damping operator. This is a continuous version of Rayleigh (or '"proportional'") damping. Also, from Eq. (12), we require

$$
\left.C_{1} \phi_{n}\right|_{x=l}=c \phi_{n}(l)+\beta E(l) A(l) \phi_{n}^{\prime}(l)=(c-\beta k) \phi_{n}(l)=0 .
$$

The presence of a spring at $x=l$ therefore necessitates a damper at $x=l$ with a particular damping coefficient $c=\beta k$ in order to give classical normal modes. For this value of $c$, the boundary damping operator becomes

$$
C_{10}=\beta k+\left.\beta E(l) A(l) \frac{\partial}{\partial x}\right|_{x=l}=\beta B_{1} .
$$

Finally, the eigenvalues $\rho_{n}, n \in \mathbf{Z}^{+}$, for the classical-damping case are given by

$$
\rho_{n}^{2}+\left(\alpha+\beta \omega_{n}^{2}\right) \rho_{n}+\omega_{n}^{2}=0 .
$$

Now consider an almost classically-damped system where for a small parameter $\epsilon$ :

$$
\begin{gathered}
\mu_{E}(x)=\alpha m(x)+\epsilon \hat{\mu}_{1}(x), \quad \mu_{l}(x)=\beta E(x)+\epsilon \hat{\mu}_{2}(x), \\
c=\beta k+\epsilon c_{1}
\end{gathered}
$$

so

$$
L_{1}=L_{10}+\epsilon L_{11}, \quad C_{1}=C_{10}+\epsilon C_{11}
$$

where

$$
\begin{gathered}
L_{11}=\hat{\mu}_{1}(x)-\frac{\partial}{\partial x}\left[\hat{\mu}_{2}(x) A(x) \frac{\partial}{\partial x}\right] \\
C_{11}=c_{1}+\left.\hat{\mu}_{2}(l) A(l) \frac{\partial}{\partial x}\right|_{x=l} .
\end{gathered}
$$

Once the various field and boundary operators are defined, the next step involves the evaluation of the functions $\mu_{p}(x)$, $v_{p}(x)$, and $h_{p}(x, t)$ in Eqs. (28), (43), and (65), respectively, if both the eigenproblem and the forced vibration problem are of interest. The function $\mu_{p}(x)$ is evaluated by applying the conditions (29) on $\partial S$,

$$
\begin{gathered}
D_{N}^{(0)} \mu_{p}=\mu_{p}=0 \quad \text { at } \quad x=0 \\
\mathcal{B}_{1 p} \mu_{p}=\zeta_{1 p} \text { at } x=l,
\end{gathered}
$$

where

$$
\begin{aligned}
\mathcal{B}_{1 p} \mu_{p} & =B_{1} \mu_{p}+\rho_{p} C_{10} \mu_{p}=\left(1+\beta \rho_{p}\right) B_{1} \mu_{p} \\
& =\left(1+\beta \rho_{p}\right)\left[k \mu_{p}(l)+E(l) A(l) \mu_{p}^{\prime}(l)\right]
\end{aligned}
$$




$$
\zeta_{1 p}=-\rho_{p} C_{11} \phi_{p}=-\rho_{p}\left[c_{1} \phi_{p}(l)+\hat{\mu}_{2}(l) A(l) \phi_{p}^{\prime}(l)\right] .
$$

Therefore, $\mu_{p}(x)$ can be chosen in the linear form,

$$
\mu_{p}(x)=c_{p} x+d_{p},
$$

with the conditions at $x=0$ and $x=l$ giving, respectively, $d_{p}$ $=0$ and

$$
c_{p}=-\rho_{p} \frac{c_{1} \phi_{p}(l)+\hat{\mu}_{2}(l) A(l) \phi_{p}^{\prime}(l)}{\left(1+\beta \rho_{p}\right)[k l+E(l) A(l)]} .
$$

This gives from Eqs. (36), (37), and (39)

$$
\begin{gathered}
\mu_{p q}=c_{p} \int_{0}^{l} x m(x) \phi_{q}(x) d x \\
\hat{\zeta}_{p q}=\left(C_{11} \phi_{p}, \phi_{q}\right)_{\partial S_{\mathrm{i}}}=\left[C_{11} \phi_{p}\right]_{x=l} \phi_{q}(l) \\
=c_{1} \phi_{p}(l) \phi_{q}(l)+\hat{\mu}_{2}(l) A(l) \phi_{p}^{\prime}(l) \phi_{q}(l) \\
=\left[c_{1}-k \hat{\mu}_{2}(l) / E(l)\right] \phi_{p}(l) \phi_{q}(l) \\
\phi_{p q}=\int_{0}^{l} \hat{\mu}_{1}(x) \phi_{p}(x) \phi_{q}(x) d x \\
-\int_{0}^{l}\left[\hat{\mu}_{2}(x) A(x) \phi_{p}^{\prime}(x)\right]^{\prime} \phi_{q}(x) d x \\
=\int_{0}^{l} \hat{\mu}_{1}(x) \phi_{p}(x) \phi_{q}(x) d x+\int_{0}^{l} \hat{\mu}_{2}(x) A(x) \phi_{q}^{\prime}(x) d x \\
+k \hat{\mu}_{2}(l) \phi_{p}(l) \phi_{q}(l) / E(l) .
\end{gathered}
$$

These parameters, together with function $\mu_{p}(x)$, permit the evaluation of all the parameters needed to determine the first-order eigenquantities $\sigma_{p}$ and $\alpha_{p q}$ from Eqs. (41), (42), and (58).

In a similar fashion, application of condition (44) results in

$$
\left.D_{N}^{(0)} v_{p}\right|_{x=0}=v_{p}(0)=0,\left.\quad \mathcal{B}_{1 p} v_{p}\right|_{x=l}=\left.\eta_{1 p}\right|_{x=l}
$$

which in turn determine $v_{p}(x)$ in the form

$$
v_{p}(x)=e_{p} x
$$

where $e_{p}$ is a known constant. This allows $v_{p q}$ in Eq. (51) to be evaluated. The quantity $\left(\hat{\eta}_{p q}-\theta_{p q}\right)$ can be evaluated as in Appendix A. The second-order eigenquantities $\tau_{p}$ and $\beta_{p q}$ can then be determined from Eqs. (56), (57), and (59).

Finally, the functions $h_{p}(x, t)$, which are needed to determine the solution of the response problem up to some prescribed order in $\epsilon$ (e.g., taking $p=0,1,2$ ) are chosen by applying conditions (66). In the present example, these conditions lead to

$$
\begin{gathered}
\left.D_{N}^{(0)} h_{p}\right|_{x=0}=h_{p}(0, t)=0 \\
\left.\mathcal{B}_{1} h_{p}\right|_{x=t}=g_{1 p}(t)= \begin{cases}g(t), & p=0 \\
-C_{11} \dot{u}_{p-1}(l, t), & p \in \mathbf{Z}^{+}\end{cases}
\end{gathered}
$$

where $g(t)$ represents an applied axial force at $x=l$. Here

$$
\begin{aligned}
&\left.\mathcal{B}_{1} h_{p}\right|_{x=l}=k h_{p}(l, t)+E(l) A(l) h_{p}^{\prime}(l, t)+\beta k \dot{h}_{p}(l, t) \\
&+\beta E(l) A(l) \dot{h}_{p}^{\prime}(l, t) \\
& C_{11} \dot{u}_{p-1}(l, t)=c_{1} \dot{u}_{p-1}(l, t)+\hat{\mu}_{2}(l) A(l) \dot{u}_{p-1}^{\prime}(l, t) .
\end{aligned}
$$

Next, choose $h_{p}$ in the form

$$
h_{p}(x, t)=\lambda_{p}(t) x+\xi_{p}(t) .
$$

Then, to satisfy the above conditions on $h_{p}$, we must have $\xi_{p}(t)$ $=0$ and

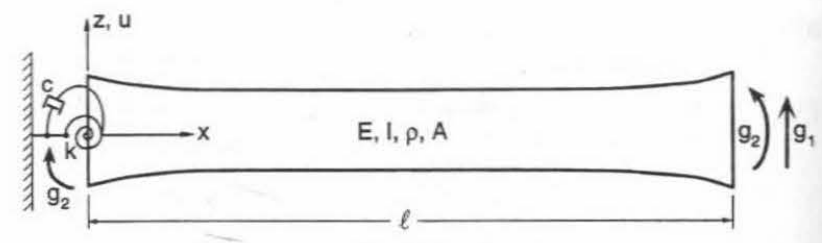

Fig. 2 Beam example

$$
\beta \dot{\lambda}_{p}(t)+\lambda_{p}(t)=\frac{1}{[k l+E(l) A(l)]} g_{1 p}(t)
$$

where $g_{10}(t)=g(t)$ and $g_{1 p}(t), p \in \mathbf{Z}^{+}$, can be expressed as

$$
\begin{aligned}
g_{1 p}(t)=-\left[c_{1}+\hat{\mu}_{2}(l) A(l)\right] \dot{\lambda}_{p-1}(t) & \\
+ & {\left[c_{1}-\frac{k \hat{\mu}_{2}(l)}{E(l)}\right] \sum_{n=1}^{\infty} \dot{q}_{p-1, n}(t) \phi_{n}(l) . }
\end{aligned}
$$

In practice, only a finite sum would be used to approximate $v_{p}(x, t)$ to sufficient accuracy and so the infinite sum in $g_{1 p}(t)$ would be replaced by a finite one. The initial condition for the differential equation for $\lambda_{p}(t)$ depends on the choice of $h_{p}(x$, 0 ), but this is arbitrary since whatever is chosen, it is corrected for by Eqs. (79) and (80). We take $h_{p}(x, 0)=0$, so $\lambda_{p}(0)=$ 0 and each $\lambda_{p}(t)$ can then be determined uniquely from a firstorder linear initial value problem. This completes the determination of the functions $h_{p}(x, t)$. The functions $h_{p n}(t)$, and so $Q_{p n}(t)$, can be evaluated from Eqs. (74) and (78) and Appendix $B$. Then, the second-order linear initial value problem given by Eqs. (77), (79), and (80) can be solved to obtain each $q_{p n}(t)$. The function $v_{p}(x, t)$ can then be approximated by the first $N$ terms in Eq. (69), where $N$ is chosen to give the desired level of accuracy. Each $u_{p}(x, t)$ is then given by Eq. (65) to finally obtain an approximation of the solution $u(x, t)$ by Eq. (60).

II: Beam Vibrations. Consider the bending vibration of the beam shown in Fig. 2, where Eq. (1) applies with $u(x, t)$ being the transverse displacement at time $t$ of a cross section at $x$ and

$$
\begin{gathered}
S=(0, l), \quad \bar{S}=[0, l], \quad \partial S=\{0, l\} \\
m(x)=\rho(x) A(x),
\end{gathered}
$$

$f(x, t)=$ distributed transverse load per unit length

$$
\begin{gathered}
L_{1}=\mu_{E}(x)+\frac{\partial^{2}}{\partial x^{2}}\left[\mu_{I}(x) I(x) \frac{\partial^{2}}{\partial x^{2}}\right], \\
L_{2}=\frac{\partial^{2}}{\partial x^{2}}\left[E(x) I(x) \frac{\partial^{2}}{\partial x^{2}}\right] .
\end{gathered}
$$

Here $m(x), I(x)$, and $E(x)$ are the mass per unit length, the cross-sectional moment of inertia, and Young's modulus at location $x$ along the beam, while $\mu_{E}(x)$ and $\mu_{I}(x)$ are non-negative viscous damping coefficients. Using Fig. 2 , the boundary conditions (2) are

$$
\begin{gathered}
D_{N}^{(0)} u=u=0 \text { and } B_{2}(0) u+C_{2}(0) \dot{u}=\mathrm{g}_{2}(0, t) \\
\text { at } x=0
\end{gathered}
$$


$B_{1}(l) u+C_{1}(l) \dot{u}=\mathrm{g}_{1}(l, t)$ and $B_{2}(l) u+C_{2}(l) \dot{u}=\mathrm{g}_{2}(l, t)$

$$
\text { at } x=l
$$

where

$$
\begin{gathered}
B_{1}(l)=-\left.\frac{\partial}{\partial x}\left[E(x) I(x) \frac{\partial^{2}}{\partial x^{2}}\right]\right|_{x=l}, \\
C_{1}(l)=-\left.\frac{\partial}{\partial x}\left[\mu_{l}(x) I(x) \frac{\partial^{2}}{\partial x^{2}}\right]\right|_{x=l} \\
B_{2}(0)=-\left.k \frac{\partial}{\partial x}\right|_{x=0}+\left.E(0) I(0) \frac{\partial^{2}}{\partial x^{2}}\right|_{x=0}, \\
C_{2}(0)=-\left.c \frac{\partial}{\partial x}\right|_{x=0}+\left.\mu_{I}(0) I(0) \frac{\partial^{2}}{\partial x^{2}}\right|_{x=0} \\
B_{2}(l)=\left.E(l) I(l) \frac{\partial^{2}}{\partial x^{2}}\right|_{x=l}, \quad C_{2}(l)=\left.\mu_{I}(l) I(l) \frac{\partial^{2}}{\partial x^{2}}\right|_{x=l}
\end{gathered}
$$

and where $g_{1}(l, t)$ and $g_{2}(0, t), g_{2}(l, t)$ are applied force and moments at the boundaries, while $c$ and $k$ are the damping and stiffness coefficients of the rotational dashpot and spring at $x$ $=0$.

Using integration by parts and the appropriate inner product, we get for all $\psi, \phi \in V \subset C^{4}(0, l) \cap C^{3}[0, l]$ satisfying the geometric boundary condition at $x=0$,

$\left\langle L_{1} \psi, \phi\right\rangle-\left\langle\psi, L_{1} \phi\right\rangle$

$$
=\left[\psi C_{1} \phi-\phi C_{1} \psi\right]_{0}^{l}+\left[\psi^{\prime} C_{2} \phi-\phi^{\prime} C_{2} \psi\right]_{0}^{l} .
$$

According to (3) with $n_{b}=2, C_{1}$ and $C_{2}$ are therefore confirmed to be compatible boundary operators for $L_{1}$. In a similar manner, $B_{1}$ and $B_{2}$ can be confirmed to be compatible boundary operators for $L_{2}$.

For the undamped problem, the frequencies and modeshapes are determined by

$$
\begin{gathered}
{\left[E(x) I(x) \phi_{n}^{\prime \prime}(x)\right]^{\prime \prime}=\omega_{n}^{2} m(x) \phi_{n}(x) \quad \text { in }(0, l)} \\
\phi_{n}(0)=0, \quad k \phi_{n}^{\prime}(0)-E(0) I(0) \phi_{n}^{\prime \prime}(0)=0 \\
\left.\frac{d}{d x}\left[E(x) I(x) \phi_{n}^{\prime \prime}(x)\right]\right|_{x=l}=0, \quad E(l) I(l) \phi_{n}^{\prime \prime}(l)=0 .
\end{gathered}
$$

We assume that this eigenvalue problem gives a countable set of eigenvalues $\left\{\omega_{n}^{2}: n \in \mathbf{Z}^{+}\right\}$and a complete set of corresponding orthonormal eigenfunctions $\left\{\phi_{n}: n \in \mathbf{Z}^{+}\right\}$.

For a classically damped system, conditions (11) and (12) must be satisfied. This will be the case if we choose

$$
\mu_{E}(x)=\alpha m(x), \quad \mu_{I}(x)=\beta E(x), \quad \beta_{n}=\alpha+\beta \omega_{n}^{2}
$$

as in Example I, and then

$$
\begin{aligned}
\left.C_{1} \phi_{n}\right|_{x=l} & =-\beta \frac{d}{d x}\left[E(x) I(x) \phi_{n}^{\prime \prime}(x)\right]_{x=l}=0 \\
\left.C_{2} \phi_{n}\right|_{x=l} & =\beta E(l) I(l) \phi_{n}^{\prime \prime}(l)=0 \\
\left.C_{2} \phi_{n}\right|_{x=0} & =-c \phi_{n}^{\prime}(0)+\beta E(0) I(0) \phi_{n}^{\prime \prime}(0) \\
& =(\beta k-c) \phi_{n}^{\prime}(0)=0
\end{aligned}
$$

if the rotational dashpot and spring at $x=0$ satisfy the condition $c=\beta k$ for classical normal modes. This means that the classical damping operators are

$$
L_{10}=\alpha m+\beta L_{2}, \quad C_{10}=\beta B_{1}, \quad C_{20}=\beta B_{2} .
$$

Now consider an almost classically damped system with:

$$
\begin{gathered}
\mu_{E}(x)=\alpha m(x)+\epsilon \hat{\mu}_{1}(x), \quad \mu_{I}(x)=\beta E(x)+\epsilon \hat{\mu}_{2}(x), \\
c=\beta k+\epsilon c_{1},
\end{gathered}
$$

So

$$
\begin{gathered}
L_{1}=L_{10}+\epsilon L_{11} \quad \text { on }(0, l) \\
C_{1}=C_{10}+\epsilon C_{11} \quad \text { at } x=l \\
C_{2}=C_{20}+\epsilon C_{21} \quad \text { at } x=0, l
\end{gathered}
$$

where

$$
\begin{gathered}
L_{11}=\hat{\mu}_{1}(x)+\frac{\partial^{2}}{\partial x^{2}}\left[\hat{\mu}_{2}(x) I(x) \frac{\partial^{2}}{\partial x^{2}}\right] \\
C_{11}(l)=-\left.\frac{\partial}{\partial x}\left[\hat{\mu}_{2}(x) I(x) \frac{\partial^{2}}{\partial x^{2}}\right]\right|_{x=l} \\
C_{21}(0)=-\left.c_{1} \frac{\partial}{\partial x}\right|_{x=0}+\left.\hat{\mu}_{2}(0) I(0) \frac{\partial^{2}}{\partial x^{2}}\right|_{x=0} \\
C_{21}(l)=\left.\hat{\mu}_{2}(l) I(l) \frac{\partial^{2}}{\partial x^{2}}\right|_{x=l} .
\end{gathered}
$$

At this point, the function $\mu_{p}(x)$ can be evaluated by applying the conditions (29) on the boundary

$$
\begin{gathered}
\mu_{p}(0)=0, \quad \mathcal{B}_{1 p} \mu_{p}=\zeta_{1 p} \equiv-\rho_{p} C_{11} \phi_{p} \quad \text { at } x=l \\
\mathcal{B}_{2 p} \mu_{p}=\zeta_{2 p} \equiv-\rho_{p} C_{21} \phi_{p} \quad \text { at } x=0, l
\end{gathered}
$$

where from (24),

$$
\mathcal{B}_{i p}=B_{i}+\rho_{p} C_{i 0}=\left(1+\beta \rho_{p}\right) B_{i} .
$$

These conditions can be satisfied by a cubic of the form

$$
\mu_{p}(x)=c_{p 3} x^{3}+c_{p 2} x^{2}+c_{p 1} x
$$

where the coefficients $c_{p 1}, c_{p 2}$, and $c_{p 3}$ are determined by the nonsingular linear system of equations

$$
\begin{gathered}
\frac{\partial}{\partial x}\left[E(x) I(x)\left(6 c_{p 3} x+2 c_{p 2}\right)\right]_{x=l}=\frac{\rho_{p}}{1+\beta \rho_{p}}\left[C_{11} \phi_{p}\right]_{x=l} \\
k c_{p 1}-2 E(0) I(0) c_{p 2}=\frac{\rho_{p}}{1+\beta \rho_{p}}\left[C_{21} \phi_{p}\right]_{x=0} \\
E(l) I(l)\left(6 c_{p 3} l+2 c_{p 2}\right)=\frac{-\rho_{p}}{1+\beta \rho_{p}}\left[C_{21} \phi_{p}\right]_{x=l} .
\end{gathered}
$$

This allows the parameters $\mu_{p q}$ in (36) to be determined. Furthermore, $\hat{\zeta}_{p q}$ and $\phi_{p q}$ in (37) and (39) are given by

$$
\begin{gathered}
\hat{\zeta}_{p q}=\left[\phi_{q} C_{11} \phi_{p}\right]_{x=l}+\left[\phi_{q}^{\prime} C_{21} \phi_{p}\right]_{x=0}^{x=l} \\
\phi_{p q}=\int_{0}^{l} \phi_{q}(x) L_{11} \phi_{p}(x) d x .
\end{gathered}
$$

These parameters allow the first-order eigenvalue correction $\sigma_{p}$ to be computed from (41) and the first-order eigenfunction correction $\chi_{p}(x)$ to be computed from (28), (32), (42), and (58).

In a similar fashion, $v_{p}(x)$ in (43) can be determined in the form

$$
v_{p}(x)=e_{p 3} x^{3}+e_{p 2} x^{2}+e_{p 1} x
$$

by the conditions (44) that it must satisfy

$$
\begin{gathered}
v_{p}(0)=0, \quad \mathcal{B}_{1 p} v_{p}=\eta_{1 p} \quad \text { at } x=l \\
\mathcal{B}_{2 p} v_{p}=\eta_{2 p} \quad \text { at } x=0, l .
\end{gathered}
$$


This allows the parameters $v_{p q}$ in (51) to be determined. The quantity $\left(\hat{\eta}_{p q}-\theta_{p q}\right)$ can be evaluated as in Appendix A. Therefore, the second-order eigenvalue correction $\tau_{p}$ can be computed from (56) and the second-order eigenfunction correction $\psi_{p}(x)$ can be computed from (43), (47), (57), and (59).

In order to determine the solution of the response problem up to, say, second order in $\epsilon$, the functions $h_{p}(x, t)(p=0,1$, 2 ) in (65) must be specified so that they satisfy the conditions (66),

$$
\begin{gathered}
h_{p}(0, t)=0, \quad B_{1} h_{p}+\beta B_{1} \dot{h}_{p}=g_{1 p} \quad \text { at } x=l \\
B_{2} h_{p}+\beta B_{2} \dot{h}_{p}=g_{2 p} \quad \text { at } x=0, l,
\end{gathered}
$$

where $g_{i p}(x, t)$ is given by (64). To satisfy these conditions, try a function of the form

$$
h_{p}(x, t)=\sum_{i=1}^{2}\left[a_{i p}(x) \lambda_{i p}(t)+b_{i p}(x) \xi_{i p}(t)\right] .
$$

It follows that

$$
\begin{gathered}
0=h_{p}(0, t)=\sum_{i=1}^{2}\left[a_{i p}(0) \lambda_{i p}(t)+b_{i p}(0) \xi_{i p}(t)\right] \\
g_{1 p}(l, t)=\sum_{i=1}^{2}\left[B_{1} a_{i p}(x)\left(\beta \dot{\lambda}_{i p}(t)+\lambda_{i p}(t)\right)\right. \\
\left.+B_{1} b_{i p}(x)\left(\beta \dot{\xi}_{i p}(t)+\xi_{i p}(t)\right)\right]_{x=l} \\
g_{2 p}(x, t)=\sum_{i=1}^{2}\left[B_{2} a_{i p}(x)\left(\beta \dot{\lambda}_{i p}(t)+\lambda_{i p}(t)\right)\right. \\
\left.+B_{2} b_{i p}(x)\left(\beta \dot{\xi}_{i p}(t)+\xi_{i p}(t)\right)\right] \quad \text { at } x=0, l .
\end{gathered}
$$

These equations are satisfied if

$$
\begin{gathered}
a_{1 p}(0)=a_{2 p}(0)=0, \quad b_{1 p}(0)=b_{2 p}(0)=0 \\
\left.B_{1} a_{1 p}\right|_{x=l}=\left.B_{1} a_{2 p}\right|_{x=l}=0,\left.\quad B_{1} b_{1 p}\right|_{x=l}=1,\left.\quad B_{1} b_{2 p}\right|_{x=l}=0 \\
\left.B_{2} a_{1 p}\right|_{x=l}=\left.B_{2} a_{2 p}\right|_{x=l}=0,\left.\quad B_{2} b_{1 p}\right|_{x=l}=0,\left.\quad B_{2} b_{2 p}\right|_{x=l}=1 \\
\left.B_{2} a_{1 p}\right|_{x=0}=0,\left.\quad B_{2} a_{2 p}\right|_{x=0}=1,\left.\quad B_{2} b_{1 p}\right|_{x=0}=\left.B_{2} b_{2 p}\right|_{x=0}=0 \\
\beta \dot{\xi}_{1 p}(t)+\xi_{1 p}(t)=g_{1 p}(l, t), \quad \beta \dot{\xi}_{2 p}(t)+\xi_{2 p}(t)=g_{2 p}(l, t) \\
\beta \dot{\lambda}_{2 p}(t)+\lambda_{2 p}(t)=g_{2 p}(0, t) .
\end{gathered}
$$

It is sufficient to set $a_{1 p}(x)=0$ on $[0, l]$ and so $\lambda_{1 p}(t)$ is not required. Also, we can take $a_{2 p}(x), b_{1 p}(x)$, and $b_{2 p}(x)$ as cubics with the four coefficients in each cubic selected to satisfy the above four conditions on each of these functions. Finally, we can take $h_{p}(x, 0)=0$ which gives the initial conditions $\xi_{1 p}(0)$ $=\xi_{2 p}(0)=\lambda_{2 p}(0)=0$. This leads to a first-order linear initial value problem for each of $\xi_{1 p}(t), \xi_{2 p}(t)$, and $\lambda_{2 p}(t)$. Solution of these three initial value problems completes the determination of the functions $h_{p}(x, t)$.

The remainder of the procedure follows the description in Example I, where $h_{p n}(t), Q_{p n}(t)$, and $q_{p n}(t)$ are determined successively and $v_{p}(x, t)$ in (69) is approximated by the first $N$ terms to give the desired level of accuracy. Each $u_{p}(x, t)$ is then given by (65) to finally obtain an approximation of the beam displacement $u(x, t)$ by $(60)$.

\section{Summary}

Conditions are presented for the existence of classical normal modes in continuous linear vibrating systems which have compatible (internal and boundary) stiffness and damping operators. A perturbation analysis is then developed for the response of almost classically damped continuous linear systems. The latter methodology provides approximate but sufficiently accurate analytical solutions for the eigenproblem as well as the response under general forcing functions.
The analysis is based on a decomposition of the internal and boundary damping operators into a part that results in classical normal mode response, with arbitrarily large damping ratios, plus a small perturbation. This permits application of standard modal analysis methodologies and provides the solution of the problem in terms of the frequencies and modes of the corresponding undamped problem only. A special treatment is presented for the series representations of the solutions in terms of the undamped eigenfunctions so that Gibbs phenomenon is avoided at the boundary. This ensures faster convergence of the eigenfunction expansions, that is, a smaller number of terms is required to give an approximation of the solutions to within a specified accuracy.

The methodology presented here is expected to result in computational benefits, especially when performing parametric studies to investigate damping effects for design purposes.

\section{References}

Bellos, J., and Inman, D. J., 1989, “A Survey of Nonproportional Damping,' Shock and Vibration Digest, Vol. 21, No. 10, pp. 7-12.

Bergman, L. A., and Nicholson, J. W., 1985, "Forced Vibration of a Damped Combined Linear System," ASME Journal of Vibration, Acoustics, Stress and Reliability in Design, Vol. 107, pp. 275-281.

Caughey, T. K., 1960, “Classical Normal Modes in Damped Linear Dynamic Systems," ASME Journal OF APPLIED MeChanics, Vol. 27, pp. 269-271.

Caughey, T. K., and O'Kelly, M. E. J., 1965, “Classical Normal Modes in Damped Linear Dynamic Systems," ASME Journal of APPLIED MECHANics, Vol. 32 , pp. $583-588$.

Chung, K. R., and Lee, C. W., 1986, “Dynamic Reanalysis of Weakly Nonproportional Damped Systems," Journal of Sound and Vibration, Vol. 111, pp. 3750.

Courant, R., and Hilbert, D., 1989, Methods of Mathematical Physics, John Wiley and Sons, New York.

Cronin, D. L., 1976, "Approximation for Determining Harmonically Excited Response of Nonclassically Damped Systems," ASME Journal of Engineering for Industry, Vol. 98, pp. 43-47.

Erdelyi, A., 1956, Asymptotic Expansions, Dover, New York.

Foss, K. A., 1958, "Coordinates Which Uncouple the Equations of Motion of Damped Linear Dynamic Systems," ASME Journal of APPLIEd Mechanics, Vol. 25 , pp. $361-364$.

Inman, D. J., and Andry, A. N., 1982, "The Nature of the Temporal Solutions of Damped Distributed Systems With Classical Normal Modes,' ASME JouRnAL. OF APplied MeChanics, Vol. 49, pp. 867-870.

Inman, D. J., 1989, Vibration with Control, Measurement and Stability, Prentice-Hall, Englewood Cliffs, NJ.

Knowles, J. K., 1985, “On Damped Linear Dynamical Systems," Ingenieur Archives, Vol. 55, pp. 321-332.

Kevorkian, J., and Cole, J. D., 1981, Perturbation Methods in Applied Mathematics, Springer-Verlag, New York.

Meirovitch, L., 1967, Analytical Methods in Vibrations, Macmillan, New York. Meirovitch, L., and Ryland, G., 1979, "Response of Slightly Damped Gyroscopic Systems," Journal of Sound and Vibration, Vol. 67, pp. 1-19.

Nashif, A. D., Jones, D. I. G., and Henderson, J. P., 1985, Vibration Damping. John Wiley and Sons, New York.

Natsiavas, S., 1993, "Dynamics of Multiple Degree of Freedom Oscillators with Colliding Components," Journal of Sound and Vibration, Vol. 165, pp. $439-453$.

Natsiavas, S., and Beck, J. L., 1994, “Almost Classically Damped Linear Discrete Systems," 12th International Modal Analysis Conference, Vol. 2, Honolulu, HI, pp. $1656-1661$.

Nicholson, D. W., 1987, "Response Bounds for Nonclassically Damped Mechanical Systems Under Transient Loads," ASME JoURNAL OF APPLIED MECHANICS, Vol. 54, pp. 430-433.

Pan, H. H., 1966, "Vibration of a Viscoelastic Timoshenko Beam," ASCE Journal of Engineering Mechanics, Vol. 92, pp. 213-234.

Plaut, R. H., and Infante, E. F., 1972, "Bounds on Motions of Some Lumped and Continuous Dynamic Systems," ASME Journal OF APPLIED MECHANics, Vol. 39, pp. 251-256.

Rayleigh, L., 1945, Theory of Sound, Vol. 1, Dover, New York.

Sharuz, S. M., and Ma, F., 1988, "Approximate Decoupling of the Equations of Motion of Linear Underdamped Systems," ASME JOURNAL OF APPLIED MECHANICS, Vol. 55, pp. 716-720.

Snowdon, J. C., 1968, Vibration and Shock in Damped Mechanical Systems, John Wiley and Sons, New York.

Udwadia, F. E., and Esfandiari, R. S., 1990, “Nonclassically Damped Dynamic Systems: An Iterative Approach," ASME JOURNAL OF APPLIED MECHANICS, Vol. 57, pp. 423-433.

Udwadia, F. E., and Kumar, R., 1994, “'Iterative Methods for Nonclassically Damped Dynamic Systems," Earthquake Engineering and Structural Dynamics. Vol. 23, pp. 137-152.

Yang, B., 1996, "Closed-Form Transient Response of Distributed Damped Systems, Part I: Modal Analysis and Green's Function Formula,"' ASME JouRNAL OF APplied Mechanics, Vol. 63, pp. 997-1003. 


\section{A P P E N D IX A}

A simplified expression is derived for the difference of $\hat{\eta}_{p q}$ and $\theta_{p q}$ which are defined in Eqs. (52) and (54), respectively. This difference is needed to evaluate the second-order eigenvalue and eigenfunction corrections, $\tau_{p}$ and $\psi_{p}(\mathbf{r})$, given in Eqs. (56), (43), (44), (47), (57), and (59).

Start by defining

$$
\begin{aligned}
& \gamma_{p q}=\sum_{i=1}^{n_{b}}\left(C_{i 0} \chi_{p}, D_{N}^{(i-1)} \phi_{q}\right)_{\partial S_{i}} \\
& \hat{\gamma}_{p q}=\sum_{i=1}^{n_{b}}\left(C_{i 1} \chi_{p}, D_{N}^{(i-1)} \phi_{q}\right)_{\partial s i} .
\end{aligned}
$$

By substituting Eq. (22) into (52), $\eta_{p q}$ can be expressed as

$$
\hat{\eta}_{p q}=-\sigma_{p} \gamma_{p q}-\rho_{p} \hat{\gamma}_{p q}-\sigma_{p} \hat{\zeta}_{p q} .
$$

Also, from Eq. (54),

$$
\begin{aligned}
\theta_{p q}= & \sigma_{p} \phi_{p q}+\left\langle\left(2 m \rho_{p} \sigma_{p}+\sigma_{p} L_{10}+\rho_{p} L_{11}\right) \chi_{p}, \phi_{q}\right\rangle \\
= & \sigma_{p}\left(2 \rho_{p}+\beta_{q}\right)\left(\alpha_{p q}+\mu_{p q}\right)+\sigma_{p} \phi_{p q} \\
& +\rho_{p}\left\langle L_{11} \chi_{p}, \phi_{q}\right\rangle-\sigma_{p} \gamma_{p q} .
\end{aligned}
$$

Here we use $\left\langle\chi_{p}, m \phi_{q}\right\rangle=\left\langle\hat{\chi}_{p}, m \phi_{q}\right\rangle+\left\langle\mu_{p}, m \phi_{q}\right\rangle=\alpha_{p q}+\mu_{p q}$, from Eqs. (28), (33), and (36), together with the compatibility Eq. (3) for $L_{10}$ and the $C_{i 0}$.

Subtracting (84) from (83),

$$
\begin{aligned}
\hat{\eta}_{p q}-\theta_{p q} & =-\rho_{p} \hat{\gamma}_{p q}-\sigma_{p}\left(\hat{\zeta}_{p q}+\phi_{p q}\right) \\
& -\sigma_{p}\left(2 \rho_{p}+\beta_{q}\right)\left(\alpha_{p q}+\mu_{p q}\right)-\rho_{p}\left\langle L_{11} \chi_{p}, \phi_{q}\right\rangle .
\end{aligned}
$$

This is the desired expression. Both $\hat{\gamma}_{p q}$ and $\left\langle L_{11} \chi_{p}, \phi_{q}\right\rangle$ can be evaluated using $\chi_{p}(\mathbf{r})=\hat{\chi}_{p}(\mathbf{r})+\mu_{p}(\mathbf{r})$ where $\hat{\chi}_{p}(\mathbf{r})$ is given by the eigenfunction expansion (32) on $\bar{S}$. In practice, only a finite number of terms, $N$, need be used in (32) to give the desired accuracy. Then $\hat{\gamma}_{p q}$ and $\left\langle L_{11} \chi_{p}, \phi_{q}\right\rangle$ can be approximated by

$$
\begin{aligned}
\hat{\gamma}_{p q}=\sum_{i=1}^{n_{b}}\left(C_{i 1} \hat{\chi}_{p}+C_{i 1} \mu_{p}, D_{N}^{(i-1)} \phi_{q}\right)_{\partial S_{i}^{i}} \\
=\sum_{n=1}^{N} \alpha_{p n} \hat{\xi}_{n q}+\sum_{i=1}^{n_{b}}\left(C_{i 1} \mu_{p}, D_{N}^{(i-1)} \phi_{q}\right)_{\partial S_{i}} \\
\left\langle L_{11} \chi_{p}, \phi_{q}\right\rangle=\left\langle L_{11} \hat{\chi}_{p}+L_{11} \mu_{p}, \phi_{q}\right\rangle \\
=\sum_{n=1}^{N} \alpha_{p n} \phi_{n q}+\left\langle L_{11} \mu_{p}, \phi_{q}\right\rangle .
\end{aligned}
$$

\section{A P P E N D I X B}

A simplified expression is derived for the $\operatorname{sum} f_{p n}(t)+\hat{g}_{p n}(t)$ required in the differential Eq. (77) for $q_{p n}(t)$ where $p, n \in$ $\mathbf{Z}^{+}$. (The $p=0$ case is relatively straightforward.) Substituting Eqs. (62) and (64) into (76) and (75), we get

$$
\begin{aligned}
f_{p n}+\hat{g}_{p n} & =-\left\langle L_{11} \dot{u}_{p-1}, \phi_{n}\right\rangle-\sum_{i=1}^{n_{b}}\left(C_{i 1} \dot{u}_{p-1}, D_{N}^{(i-1)} \phi_{n}\right)_{\partial s_{i}} \\
& =-\left\langle\dot{u}_{p-1}, L_{11} \phi_{n}\right\rangle-\sum_{i=1}^{n_{b}}\left(D_{N}^{(i-1)} \dot{u}_{p-1}, C_{i 1} \phi_{n}\right)_{\partial s_{i}}
\end{aligned}
$$

where the compatibility condition (3) for $L_{11}$ and the $C_{i 1}$ has been used. Equation (65) can now be used where $v_{p-1}(\mathbf{r}, t)$ is approximated by a finite number of terms, $N$, in the eigenfunction expansion (69), so that

$$
\begin{aligned}
f_{p n}+\hat{g}_{p n}= & -\sum_{m=1}^{N} \dot{q}_{p-1, m}\left(\phi_{n m}+\hat{\zeta}_{n m}\right)-\left\langle\dot{h}_{p-1}, L_{11} \phi_{n}\right\rangle \\
& -\sum_{i=1}^{n_{b}}\left(D_{N}^{(i-1)} \dot{h}_{p-1}, C_{i 1} \phi_{n}\right)_{\partial S_{i}}
\end{aligned}
$$

where the definitions for $\hat{\zeta}_{n m}$ and $\phi_{n m}$ in (37) and (39) have been used. Equation (88) shows that the $q_{p n}(t)$ must be evaluated sequentially with respect to increasing $p$ since $q_{p n}$ depends on the $q_{p-1, m}, m=1,2, \ldots, N$. 